\title{
El periodismo deportivo condicionado por la gestión comunicativa de los clubes de fútbol: el caso del FC Barcelona
}

\author{
Fernando Olabe SÁNCHEZ \\ Universidad Miguel Hernández de Elche \\ molabe@umh.es
}

Recibido: 30 de octubre de 2014

Aceptado: 16 de junio de 2015

\begin{abstract}
Resumen
Las relaciones entre los gabinetes de Comunicación de los clubes de fútbol y los periodistas deportivos se enmarcan en el modelo de Gieber y Johnson (1961) por el que el hecho de que ambos compartan objetivos comunes, donde los gabinetes de Comunicación necesitan que los medios publiquen determinadas informaciones y los periodistas precisan de noticias que publicar, provoca una pérdida de independencia por parte de los periodistas, ya que necesitan a esos departamentos como fuentes. En la actualidad, los departamentos de Comunicación de los clubes de fútbol, como el del FC Barcelona, se han constituido en gatekeepers. Esto ha acentuado las históricas diferencias que existen entre los periodistas y los profesionales de la comunicación corporativa, incrementado por el control informativo de estos departamentos lo que provoca constantes tensiones entre ambos.
\end{abstract}

Palabras clave: comunicación corporativa, gabinetes de prensa, medios de comunicación, periodistas deportivos, FC Barcelona.

\section{Sports journalism conditioned by the communicative management of football clubs: the case of FC Barcelona}

\begin{abstract}
Relations between press offices football clubs and sports journalists are part of the model Gieber and Johnson (1961) by the fact that both share common goals, where communication cabinets need the media to print certain information and news reporters need to publish results in loss of independence on the part of journalists, as they need to those departments as sources. Currently, the departments of Communication football clubs such as FC Barcelona, have become gatekeepers. This has accentuated the historical differences between journalists and corporate communications professionals, plus the information control of these departments causing ongoing tensions between the two.
\end{abstract}

Keywords: corporate communications, press offices, media, sports journalists, FC Barcelona.

\section{Referencia normalizada}

OLABE SÁNCHEZ, Fernando (2016): "El periodismo deportivo condicionado por la gestión comunicativa de los clubes de fútbol: el caso del FC Barcelona". Estudios sobre el Mensaje Periodístico. Vol. 22, Núm. 1 (enero-junio), págs.: 463-481. Madrid, Ediciones Complutense.

Sumario: 1. Introducción. 2. Metodología; 2.1. Revisión bibliográfica y hemerográfica; 2.2. Cuestionario a los periodistas deportivos; 2.3. Entrevistas a directivos de comunicación del FC Barcelona. 3. Las relaciones entre los clubes de fútbol, los medios de comunicación y los periodistas deportivos. 4. Los clubes de fútbol como gestores de la información periodística. 5. El modelo comunicativo del FC Barcelona. 6. Resultados; 6.1. La atención informativa sobre el FC Barcelona; 6.2. La búsqueda de la información sobre el FC Barcelona; 6.3. Fuentes no institucionales de los periodistas deportivos; 6.4. Tipo de comunicación que realiza el club. 7. Conclusiones. 8. Referencias bibliográficas. 


\section{Introducción}

El tipo de comunicación que una organización mantiene con sus públicos objetivos refleja, según Lydenberg (2005) el nivel de compromiso que desarrolla la organización con esos públicos. Por ello, coincidimos con Hopwood (2007: 34) en que actualmente se ha incorporado un nuevo paradigma a la gestión comunicativa en los clubes de fútbol, que supera la convicción de que el papel de los profesionales de la comunicación en estas entidades deportivas se circunscribe al de mantener únicamente relaciones con los medios para que estos publiquen noticias positivas. En este sentido, la relación entre los clubes y los medios también afectan a otros públicos de la entidad, como los seguidores y los patrocinadores (Ginesta, 2010: 148-149). No obstante, los clubes de fútbol entienden que las relaciones con los medios de comunicación son tan importantes para su gestión empresarial como los vínculos que establecen con los socios de esas entidades, así como con los seguidores (Cleland, 2009: 421).

Resulta evidente que los medios de comunicación contribuyen de forma significativa a la percepción que los públicos objetivos de las organizaciones deportivas adquieren sobre estas, lo que otorga un papel fundamental al departamento de Comunicación y a las acciones de Relaciones Públicas que llevan a cabo para contribuir a forjar esa imagen (Desmarais y Bruce, 2008: 1). Siguiendo a Post et al. (2002), podemos afirmar que los clubes de fútbol profesionales, como organizaciones, y los medios de comunicación, como stakeholders, poseen intereses comunes: los primeros emitir mensajes que lleguen al resto de sus públicos objetivos; los segundos, obtener información que les permita desarrollar su actividad profesional y empresarial. Se trata de una relación simbiótica, en la que ambos obtienen beneficios de su contacto, ya que tanto los medios como las entidades deportivas son instituciones sociales que tienen como objetivo el negocio (Helland, 2007: 107-111). Según este autor, la mejor ejemplificación de esa dependencia y beneficio mutuo se observa en cómo ha contribuido el deporte al desarrollo de la televisión y cómo los operadores televisivos no sólo han incrementado la visibilidad de las disciplinas deportivas, sino también su capacidad para generar ingresos atípicos.

A juicio de Hopwood (2010), la comunicación actual en los clubes de fútbol se entiende desde un modelo integral, denominado Relationship Management in Sport o enfoque relacional, que gestiona las relaciones con los públicos de interés de los clubes a través de estrategias complementarias: desde el issues management hasta la comunicación de crisis, pasando por las relaciones institucionales con los medios de comunicación. Para Ledingham (2003: 194), con la aplicación de este modelo, las organizaciones consiguen relacionarse de forma más efectiva con sus públicos objetivos, apoyándose en la comunicación como herramienta estratégica para hacer frente al compromiso que le demandan tanto sus stakeholders, como la sociedad en general.

\section{Metodología}

A la hora de diseñar la metodología empleada en nuestra investigación, nos hemos apoyado en el trabajo de Cleland (2009), que analizó las relaciones que cuatro clubes de la Premier League inglesa mantenían con los medios de comunicación. Este autor se basó en una doble metodología cuantitativa y cualitativa: a través de entrevistas 
semiestructuradas con directivos de los clubes y periodistas, así como mediante cuestionarios dirigidos a los seguidores de esos clubes.

Por tanto, para llevar a cabo este trabajo se ha optado por una metodología cuantitativa y cualitativa. Se han empleado diferentes herramientas: a) cuestionarios estructurados con preguntas cerradas dirigidas a los periodistas deportivos que siguen la información del FC Barcelona, b) entrevistas en profundidad a profesionales que han trabajado en los departamentos de Comunicación de ambos clubes y c) una investigación empírica secundaria con fuentes bibliográficas y fuentes hemerográficas.

\subsection{Revisión bibliográfica y hemerográfica}

En primer lugar, apoyándonos en la Grounded Theory, (Glaser, 1992: 16), se ha revisado la literatura académica de las principales revistas relacionadas con el management en el deporte, en las que se han buscado los ítems que nos interesan a través de las siguientes palabras clave: comunicación corporativa en el fútbol, relaciones públicas en el fútbol, gestión comunicativa en el fútbol, FC Barcelona, stakeholders en el fútbol, periodismo deportivo. En cuanto a las fuentes hemerográficas, recurrimos a las hemerotecas online de los diarios El País, ABC, Mundo Deportivo y La Vanguardia, ya que su consulta en abierto agilizaba la investigación el acceso directo a documentos, en muchos casos en formato PDF.

\subsection{Cuestionario a los periodistas deportivos}

El siguiente paso fue la elaboración de un cuestionario que posibilitara comparar y medir las necesidades informativas de los periodistas especializados en Deportes, en función del soporte en el que desarrollen su trabajo. La aportación de los cuestionarios permite la comparabilidad de las respuestas y ofrece mediciones cuantitativas de una gran variedad de aspectos objetivos y subjetivos. De este modo, pretendíamos analizar las percepciones que los periodistas, como público estratégico de los clubes de fútbol, tienen de su gestión comunicativa.

Nos hemos apoyado en los trabajos de Johnson y Weaver (2007) y de Tilley y Hollings (2008) a la hora de elaborar los cuestionarios y las preguntas dirigidas a los periodistas que habitualmente cubren informativamente la actualidad del FC Barcelona. Asimismo, hemos tenido como referencia el informe "La Imagen del Dircom. La percepción del Director de Comunicación entre los Periodistas del sector económico/financiero", un estudio sobre las relaciones entre los periodistas especializados en información económica y los directores de comunicación españoles, realizado en 2010 por la consultora de Comunicación Grayling.

Dicho informe estudia la percepción de los periodistas sobre la actividad que desarrollan los departamentos de Comunicación, en este caso de empresas del ámbito de la economía; y por otro, el objeto de estudio coincide con el nuestro: periodistas especializados y responsables de comunicación de un sector productivo concreto ${ }^{1}$.

1 El informe completo está accesible en la url: http://mouriz.files.wordpress.com/2010/05/informedircom2009.pdf 
El criterio de selección de la muestra se ha basado en aquellos periodistas que diariamente informan únicamente del club, con una relación específica con las fuentes y los diversos actores de la entidad, no incluyendo en esta categoría a los redactores jefe, jefes de sección, columnistas de opinión y otros cargos, que abordan tanto la actualidad de estos clubes como otras disciplinas o entidades. La excepción, en este caso, se refiere a los diarios especializados, en los que estos cargos directivos sí se encuentran en constante contacto con la entidad, ya que dirigen secciones específicas relacionadas con el FC Barcelona (Mundo Deportivo y Sport).

Teniendo en cuenta lo anterior, se trata de un universo poblacional reducido, compuesto por los medios generalistas y especializados de Barcelona. Los medios de comunicación se seleccionaron de acuerdo con el listado que recoge la Agenda de la Comunicación del Gobierno de España, y se tuvo en cuenta la particularidad de la estructura empresarial en esta comunidad autónoma, en la que conviven medios autóctonos con delegaciones de medios de comunicación de ámbito nacional.

Se remitieron cuestionarios a los periodistas de las emisoras de radio COM Radio, Catalunya Radio, Cadena SER, Cadena COPE, Onda Cero, Punto Radio, RNE y Radio MARCA. Por lo que respecta a los medios impresos (especializados y generalistas), se envió a periodistas de Sport, Mundo Deportivo, AS, Marca, El 9 Esportiu de Catalunya, Avui, El Periódico de Catalunya, La Vanguardia, El País, El Mundo, ABC, La Razón, Agencia EFE y Europa Press. En cuanto a los medios televisivos, se contactó con reporteros de TV3, Antena 3, Cuatro, La Sexta, TVE y Tele5.

Previamente a la elaboración del cuestionario, se realizó un pretest con periodistas de distintos soportes (televisión, prensa escrita, radio y agencia de información general) para corregir las posibles deficiencias de redacción de las preguntas y comprensión de las cuestiones, así como la idoneidad y utilidad de las variables seleccionadas para la investigación (Grande y Abascal, 2005: 41).

Los periodistas consultados en el test previo sugirieron modificar la redacción de varias preguntas para facilitar una mejor comprensión de los enunciados. Se diseñó un cuestionario semiestructurado, autoadministrado a través del acceso a una página web específica a la que remitía el enlace que se envió por correo electrónico a los periodistas seleccionados. El cuestionario, con preguntas cerradas, contenía 36 variables con tres tipos de respuesta: a) dicotómicas, b) de opción múltiple y c) de respuesta múltiple.

Se envió el cuestionario a 58 periodistas que informan sobre el FC Barcelona. La tasa de respuesta de los periodistas que informan sobre el FC Barcelona fue del 59\%. El porcentaje se sitúa entre diez y veinte puntos por encima de los registrados en estudios similares en los que se ha entrevistado a los periodistas como público objetivo (Rodríguez, 2003; Ramírez, 1995; Micó-Sanz y González-Molina, 2010). En la esfera internacional las investigaciones que han utilizado encuestas a periodistas deportivos reflejan una tasa de respuesta entre el 13\% (Wigley y Meirick, 2008) y el 21\% (Schultz y Sheffer, 2010).

Una vez obtenidos los datos, se analizaron los resultados de ambos cuestionarios, a través de un estudio estadístico descriptivo. Se efectuó un análisis univariante, con variables categóricas ordinales, tomando las frecuencias relativas, que se han expre- 
sado en porcentajes. Se pretendía describir el comportamiento general de los encuestados para cada uno de los aspectos recogidos en el cuestionario. Por último, se utilizó como parámetro estadístico el de la moda. Este criterio nos ayudó resolver las no-respuestas parciales, es decir, las preguntas que alguno de los encuestados dejó de contestar. Para ello, hemos optado por introducir un elemento corrector con la imputación de estas no-respuestas a otros estratos utilizando la medida estadística de tendencia central, en este caso la moda de las respuestas, como valor más frecuente, de todos los encuestados que han respondido (Grande y Abascal, 2005: 86), ya que los encuestados comparten su pertenencia al mismo grupo de análisis, el de los periodistas deportivos.

\subsection{Entrevistas a directivos de comunicación del FC Barcelona}

La entrevista en profundidad como instrumento de observación se realizó a uno de los responsables de comunicación del FC Barcelona, Toni Ruiz, que han desempeñado estas funciones en los últimos 15 años, con el objetivo de conocer determinados aspectos de la gestión comunicativa del FC Barcelona. Siguiendo el trabajo de Hachigian y Hallahan (2003), articulamos parte de las cuestiones de la entrevista sobre la percepción de los periodistas deportivos acerca del papel que desempeñan los departamentos de Comunicación de los clubes de fútbol como fuente de información, y también de las herramientas comunicativas que emplean estos departamentos en su relación con los periodistas deportivos.

\section{Las relaciones entre los clubes de fútbol, los medios de comunicación y los pe- riodistas deportivos}

Las relaciones entre los periodistas deportivos y los clubes como fuentes de información han evolucionado de la misma forma que el fútbol ha pasado de ser una mera competición a convertirse en un espectáculo deportivo, en el que confluyen variados intereses y públicos diversos. En los años cincuenta y sesenta, las relaciones entre los periodistas deportivos y los clubes se regían por lo que Hopcraft (2013: 188) describió como una combinación amistosa entre el día a día y el agrado profesional, basado en un contacto relajado entre ambas partes. En ese contexto, incluso, se silenciaban determinados escándalos de los clubes para no desagradar a estas fuentes informativas (Sudgen y Tomlinson, 2007: 45). No obstante, esta relación amigable que buscan los periodistas deportivos prosigue hoy día, pero se ha ampliado a otros públicos objetivos, como los propios deportistas o los patrocinadores, para no perderlos como fuentes informativas (Rowe, 2005: 132-134).

De acuerdo con Cleland (2009: 419-420) las relaciones entre los medios y los clubes de fútbol se basan, históricamente, en la interdependencia entre ambos. El interés de la opinión pública por conocer la actualidad futbolística incrementaba las ventas de los periódicos y ayudaba a los clubes a relacionarse con los seguidores. Esa dependencia, según Vinnai (1986: 73), incrementó la cobertura del fútbol como elemento prioritario en los medios de comunicación en detrimento de otras especialidades deportivas, circunstancia que se sigue manteniendo hoy día (González Ramallal, 2004: 271-280). De hecho, los gabinetes de Comunicación han adquirido en los últimos 
tiempos las mismas rutinas de producción que los medios, lo que favorece, por un lado, el trabajo de los periodistas que cubren a las organizaciones y, por otro, consiguen que su organización adquiera protagonismo mediático (Manning, 2001: 67).

En este sentido, Tomlinson y Sugden (2008: 149) sostienen que las funciones de los responsables de la comunicación de los clubes se ciñen a tres: gestionar la comunicación del club con los medios, crear noticias positivas sobre su empresa y estar atentos a aquellos periodistas críticos con la gestión del club. Según Trosby (2010: 55), la experiencia profesional como periodistas de muchos de los responsables de estos departamentos determina que la principal función se base en las relaciones con los medios.

Esta idea se reafirma cuando acudimos a Sallot y Johnson (2006: 154). En su ingente trabajo sobre cómo han evolucionado las relaciones entre periodistas y departamentos de Comunicación en Estados Unidos durante 15 años, concluyeron que para los periodistas es importante mantener una buena relación con los gabinetes de Comunicación de las organizaciones, aunque también admitían que les interesa más a los propios departamentos de Comunicación mantener esa sintonía con los medios. Las consecuencias de esta actuación resultan evidentes. Los periodistas deportivos admiten que los departamentos de Comunicación de los clubes de fútbol entregan tanto material informativo que no es necesario salir a buscar las historias con las que componer sus artículos (Boyle, 2007: 7). Estos hechos confirman, además, otra realidad: en la mayoría de las informaciones deportivas, el uso de fuentes se limita generalmente a una, incluso en muchos casos ninguna, y la información apenas se contrasta con otras fuentes (Horky, 2009: 75).

Se ha afirmado que el papel de los gabinetes de Comunicación, independientemente de que sean percibidos como fuente de información para el periodista o como fuente interesada, condiciona el trabajo de los periodistas (Ramírez, 1995: 53). Una de las consecuencias es que se produce un mayor control y acceso a la información que generan los clubes y los deportistas, eliminando los contactos informales que en otras épocas mantenían estos con los periodistas (Sugden y Tomlinson, 2007). Esta situación ha influido en el desarrollo del periodismo deportivo, según Boyle y Haynes (2009: 177), ya que los periodistas disponen de mayor acceso a la información suministrada por los departamentos de Comunicación, pero al mismo tiempo, dicho acceso les limita en la búsqueda de sus propios temas y a la hora de profundizar sobre ellos.

Los periodistas, actualmente, ya no controlan de la misma forma que antaño tanto lo que se trasmite como lo que llega a las audiencias, debido no solo al desarrollo de los gabinetes de Comunicación, sino a las posibilidades que les ofrecen a los clubes de fútbol las nuevas tecnologías que les permiten relacionarse con sus públicos sin necesitar la intermediación de los periodistas (White y Hobsbawn, 2007: 286).

A esto se añaden otros condicionantes. Aunque es evidente y notorio que, desde sus inicios, la relación entre las organizaciones deportivas y los medios de comunicación ha sido de mutuo interés, y que los medios impresos han sido el gran referente para clubes e instituciones deportivas (Lever y Wheeler, 1993: 129), la influencia de la televisión (Rowe, 1996: 570) y de los nuevos medios sociales ha modificado el comportamiento de ambos protagonistas del negocio del deporte (Schultz y Sheffer, 2010: 235). Al mismo tiempo, en los últimos años se ha incrementado la supervisión sobre 
la información que generan los clubes y los jugadores por parte de los departamentos de Comunicación de esas entidades, para de esa forma impedir las relaciones informales que anteriormente se producían entre los jugadores y los periodistas deportivos (Boyle, 2007: 7).

Podemos concluir, por tanto, que el modelo comunicativo de los clubes de fútbol está cambiando, favorecido por la inclusión en su estrategia comunicativa de las TCI, lo que les conduce a la coexistencia de los modelos de comunicación offline y online, en mutua convivencia (Cleland, 2009: 417). La entidad ha pasado de ser fuente a ser editora de las propias noticias que genera, por lo que el papel de los medios de comunicación se ha desdibujado. La puesta en marcha de nuevos soportes (webs corporativas, canales de televisión, twitter, blogs o radios), que se suman a las tradicionales publicaciones impresas con las que mantenían en el pasado su relación con los aficionados, ha generado un nuevo ecosistema informativo en torno a la actualidad de estas entidades deportivas, un espacio que ya no está dominado por los medios tradicionales, que han perdido su hegemonía al alterarse los polos de emisión (Prudkin, 2010: 7-9).

Al mismo tiempo, en paralelo a este cambio de roles, han surgido nuevas fuentes en el ámbito de la información deportiva, que obligan a los actores principales (clubes de fútbol y medios) a modificar su comportamiento comunicativo: los jugadores y su uso de las redes sociales, que conectan directamente con los seguidores sin la intermediación ni de los medios tradicionales ni de los canales comunicativos de las entidades deportivas. Al mismo tiempo, los clubes de fútbol también han de adecuar los contenidos de sus propios medios, ya que sus seguidores constituyen una audiencia fragmentada, que emplea diversos medios y soportes comunicativos para recibir la actualidad informativa relacionada con su club (Kuntz et al., 2010: 21).

\section{Los clubes de fútbol como gestores de la información periodística}

El escenario global actual ha provocado importantes cambios en la actuación de los departamentos de Comunicación de los clubes de fútbol: por un lado, ha permitido integrar las nuevas herramientas comunicativas que aportan las TCI y que posibilitan diferentes maneras de relacionarse con los públicos de estas entidades (Boyle y Haynes, 2009); por otro, como consecuencia de ello, se han superado los modelos comunicativos tradicionales que empleaban estos departamentos, basados principalmente en las relaciones informativas con los medios tradicionales (Schultz et al., 2010). Los clubes han pasado a crear y gestionar sus informaciones a través de una multiplataforma de medios propios, con el objetivo de reducir su dependencia de las informaciones editadas por los medios tradicionales (Moragas, et al., 2011).

Por tanto, los departamentos de Comunicación de los clubes de fútbol se han constituido, impulsados por las nuevas tecnologías de la Comunicación y la Información, en productores de información que gestionan ellos mismos para controlar los mensajes a sus públicos objetivos sin la necesidad de la intermediación comunicativa de los medios de comunicación. Esta realidad se traduce en los clubes de fútbol, ya que la cobertura va más allá de los tradicionales eventos deportivos, como los partidos correspondientes a las competiciones en las que participa, y se amplía a otras situacio- 
nes noticiosas que el club gestiona a través de sus propios soportes comunicativos, desde las webs corporativas hasta la televisión y radio del club o las publicaciones impresas (Ginesta, 2010).

Cabe recordar que hasta la aparición de los medios propios de los clubes de fútbol, el debate sobre quién establecía la agenda se centraba en que los contenidos de los medios no solo provenían de sus rutinas productivas, sino también de las aportaciones provenientes de los departamentos de Comunicación de las instituciones, que desarrollaban estrategias proactivas, basadas en técnicas de Relaciones Públicas, para conseguir que sus mensajes estuvieran no solo en la agenda de los medios sino también en la de los públicos (Fortunato, 2001: 484).

El control sobre el contenido de la agenda relativo al fútbol en España ya no queda en manos de los medios, como ha sido tradicional (Golan y Wanta, 2001), donde los departamentos de Comunicación utilizaban a los medios para hacer llegar sus mensajes a su mapa de públicos (Hallahan, 1999: 238). En la actualidad, los mensajes los controlan los departamentos de Comunicación de los clubes a través de sus propios soportes informativos (Ginesta, 2010), por lo que ya no son necesarias las distintas herramientas propias de la comunicación corporativa para conseguir construir la agenda pública (Curtin, 1999).

El nuevo modelo comunicativo de los clubes de fútbol, basado en dotar de mayor relevancia a la gestión de sus propios soportes comunicativos para controlar la información que genera, entronca con lo que Matilla (2010: 10) entiende como nueva corriente: "La filosofía basada en la naturaleza y la calidad de las relaciones con el mapa de públicos es sustituida por la de la rentabilidad contable de los resultados de las inversiones en comunicación". Pese al cambio en la estrategia que supone que los gabinetes de Comunicación de los clubes de fútbol se hayan constituido en productores de noticias a través de sus propios soportes informativos, siguen necesitando la cobertura mediática de sus partidos (Desmarais y Bruce, 2008: 188-189) y de buena parte de las noticias que generan estos encuentros, por lo que los departamentos de Comunicación utilizan las herramientas de la comunicación institucional que faciliten el trabajo de los periodistas (Stoldt et al., 2011: 283).

De hecho, Desmarais y Bruce (2008: 184) consideran que mediante esta cobertura mediática, especialmente la audiovisual, los medios también contribuyen a difundir la imagen de marca, por lo que, en cierto modo, se les puede calificar de "relaciones públicas" de los clubes. No hay que olvidar que en el caso de los clubes españoles, los propios departamentos de comunicación de estas entidades reconocen que sus principales públicos objetivos son los abonados, los medios de comunicación y los patrocinadores (Sanahuja, 2013).

\section{El modelo comunicativo del FC Barcelona}

El club catalán concita buen parte de la atención informativa e interés mediático, que se ha ido incrementado en los últimos tiempos en lo que se ha calificado de centrismo y superabundancia informativa (González Ramallal, 2014: 348), al tener un seguimiento informativo durante las 24 horas del día. Para generar ese interés, el funcionamiento del departamento de Comunicación del FC Barcelona se desarrolla en tres ámbitos, condi- 
cionado por la constante atención mediática que recibe la entidad, tanto en España como en la esfera internacional, según Toni Ruiz, jefe de prensa de la entidad².

- Dar respuesta a las peticiones de los medios. Muchas veces, el departamento de Comunicación actúa como filtro, sobre todo cuando se refiere al primer equipo de fútbol, ya que tiene que atender un elevado número de peticiones de entrevistas y reportajes de diferentes medios.

- Como departamento de Comunicación al uso. Cuando se ha de transmitir información que no es estrictamente deportiva, sino relativa a la gestión de las otras secciones del club, de la fundación o de la propia institución. El departamento de Comunicación no solo atiende a los periodistas que siguen la actualidad del primer equipo de fútbol, sino que también ha de gestionar la información que genera la actividad de las secciones de baloncesto, balonmano, hockey y fútbol sala, la del fútbol formativo, a la prensa internacional que se preocupan por el fenómeno y los proyectos generados por el departamento comercial y la fundación.

- La redacción de contenidos. El departamento de Comunicación ha diseñado una estrategia para dar cobertura a las necesidades de los medios y dotar de contenidos exclusivos a sus públicos estratégicos, a través de una redacción propia que prepara contenidos diariamente para distribuir a socios, a aficionados y también a los medios, "porque actuamos de agencia, a través de diferentes medios de comunicación", asevera Ruiz.

Según el ex director de Comunicación del club Jordi Badía (2009: 96), los medios se resignaron a aceptar que el club utilizara sus propios soportes comunicativos, como Barça TV, para comunicar ciertos contenidos que preferían no exponer a través de los soportes tradicionales de la comunicación institucional como ruedas de prensa o comunicados de prensa, especialmente cuando se trataba de una estrategia en la que quedaba de manifiesto la voluntad de ser la única fuente ante socios y seguidores, sin que mediara la interpretación del periodista. No obstante, buena parte de los periodistas deportivos consideran que el hecho de que el Barça controle y gestione la información de la actualidad del club se ha convertido en un monopolio y entienden que ese modelo de gestión comunicativa empobrece la marca y la reputación del club (Santos, 2010: 52).

Ante ello, desde el departamento de Comunicación se insiste en su voluntad de no convertirse en un competidor con los diferentes medios, y se asegura que constantemente se busca el equilibrio entre las necesidades informativas de los periodistas y los intereses corporativos de la entidad. Para los responsables de Comunicación del FC Barcelona, las críticas de los periodistas deportivos sobre el control de la información que realiza el club se deben contextualizar en el fenómeno de la profesionalización de un deporte que ha trascendido su carácter meramente deportivo para situarse en un plano puramente empresarial, tal y como advierte Ruiz:

"Cuando digo que hay un ejercicio constante de equilibrio con los medios de comunicación me refiero a que, por una parte, el sector es consciente de la evolución de la tecnología y el desarrollo de los medios que antes no existían y que hoy están al alcance de cualquiera, incluso de las instituciones como nosotros que generan contenido; y por otra parte, también entienden la necesidad del club como tal de tener una voz propia, porque al

${ }^{2}$ Entrevista personal mantenida en la sede del club. 
final cualquier empresa importante, de un sector importante, necesita tener voz propia, sobre todo cuando se dirige a un público concreto".

Para Toni Ruiz, la credibilidad del departamento de Comunicación ante los periodistas se basa en que estos confien en que no se les va a mentir. Por eso, sostiene que "es preferible no responder en los casos conflictivos para el club, antes que dar una respuesta que pueda generar equívocos".

También se ha de tener en cuenta que este comportamiento muestra la evolución del modelo comunicativo del FC Barcelona. De una época en la que la atención informativa sobre el club no estaba masificada, y en la que los periodistas departían de tú a tú con los jugadores y técnicos, estableciendo incluso relaciones personales entre ellos, se pasa al momento actual en el que los clubes blindan a los jugadores ante los medios de comunicación (Barroso, 2012). Esta situación ha generado, según Toni Ruiz, una deshumanización de esas relaciones, ya que los jugadores no conocen a los periodistas. La ingente cantidad de medios de comunicación que siguen la actualidad del FC Barcelona tiende a aumentar las demandas sobre los protagonistas de la información.

En este punto, surge la comparativa con el modelo comunicativo implantado en otras ligas de fútbol europeas, como la inglesa, donde los equipos entrenan semanalmente a muchos kilómetros de distancia de las ciudades, con el propósito de aislar a los jugadores y técnicos de la atención informativa de los medios de comunicación (Boyle, 2010). Para Toni Ruiz (2011), esta dinámica de la Premier League inglesa favorece a los clubes, hasta el punto de que admite que en esa situación no sería preciso un departamento de Comunicación que coordinara las relaciones entre los medios y los jugadores, ya que los periodistas deportivos seguirían sin poder asistir a los entrenamientos.

Por la propia dimensión del club, el interés informativo de la institución no sólo se centra en la esfera deportiva. El departamento de Comunicación también debe atender las peticiones de medios de otros ámbitos informativos, como la prensa económica, la internacional o la social. De ahí que desde el departamento de Comunicación se implementen contenidos específicos, desde una actitud proactiva de la información, en la que la actividad que desarrolla la Fundación del FC Barcelona y sus acciones de Responsabilidad Social Corporativa adquiere un papel determinante.

\section{Resultados}

\subsection{La atención informativa sobre el FC Barcelona}

El nivel de interés informativo que genera el FC Barcelona se aprecia por la frecuencia de las relaciones que los periodistas mantienen con la entidad. El $94 \%$ de los periodistas contacta diariamente con el club, de los cuales un 56\% lo hace varias veces al día, lo que pone de manifiesto que la relación se amplía más allá de los actos que convoca la institución (ruedas de prensa, entrenamientos, entrevistas, etc.).

\subsection{La búsqueda de la información sobre el FC Barcelona}

\section{a) Las fuentes institucionales del club}

A la hora de realizar sus informaciones, los periodistas emplean una amplia diversidad de fuentes que dotan de contenidos sus informaciones. En el caso del FC Bar- 
celona, se aprecia que los medios propios del club se han convertido en una fuente informativa diaria para los periodistas deportivos: un $79 \%$ acude a las redes sociales oficiales, un 56\% lo hace al sitio web corporativo y un $35 \%$ se nutre de contenidos de Barça TV. El gabinete de prensa del FC Barcelona también se ha constituido en un referente informativo para los periodistas catalanes, ya que un $41 \%$ de ellos contacta varias veces al día con este departamento.

El presidente del club también ha adquirido el estatus de fuente informativa para los periodistas deportivos, aunque sin una periodicidad determinada: un $56 \%$ en el caso del presidente del FC Barcelona. Se destaca, no obstante, que para un 26\% de los periodistas catalanes, el presidente del Barça no es fuente informativa.

También son fuentes atemporales los directivos (para un 50\% de los periodistas), el director de Comunicación del club (para un 41\%), así como los protagonistas directos de la información deportiva: los jugadores (41\%) y el cuerpo técnico del primer equipo (38\%).

\section{b) Credibilidad de las fuentes institucionales del club}

El gabinete de prensa del FC Barcelona es la fuente institucional que más credibilidad ofrece para los periodistas deportivos: el 71\%) de los encuestados confían en él. Otras fuentes institucionales que también les dan garantías a los informadores catalanes son los jugadores $(68 \%$ ) y los medios propios de la entidad: las redes sociales oficiales (68\%), el sitio web corporativo (65\%) y la televisión del club (65\%). También los directivos constituyen una fuente institucional bastante o totalmente creíble para un $62 \%$ de los encuestados. En menor medida, los periodistas confían en el presidente (56\%), en el director de Comunicación (56\%) y en el cuerpo técnico del primer equipo (56\%).

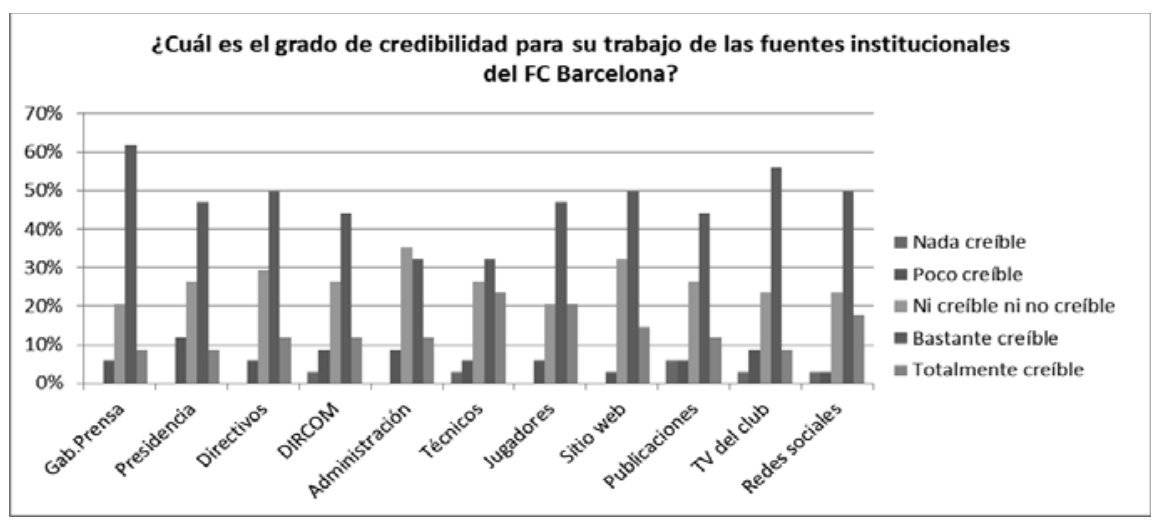

Gráfico 1. Credibilidad para los periodistas de las fuentes institucionales del FC Barcelona. Fuente: elaboración propia

\section{c) Utilidad de las fuentes institucionales del club}

$\mathrm{Si}$ ahondamos en el comportamiento de los periodistas que cubren la actualidad del FC Barcelona, detectamos que un 79\% de ellos considera que los jugadores son la fuente más útil en sus rutinas productivas y para el $70 \%$ el cuerpo técnico también es una fuente necesaria para su trabajo. Para el $68 \%$ de estos periodistas deportivos, el gabinete de prensa desempeña un papel relevante en su trabajo, así como las redes so- 
ciales oficiales del club, que son útiles para el $68 \%$ de los informadores. También es útil el sitio web corporativo para un $65 \%$ de ellos.

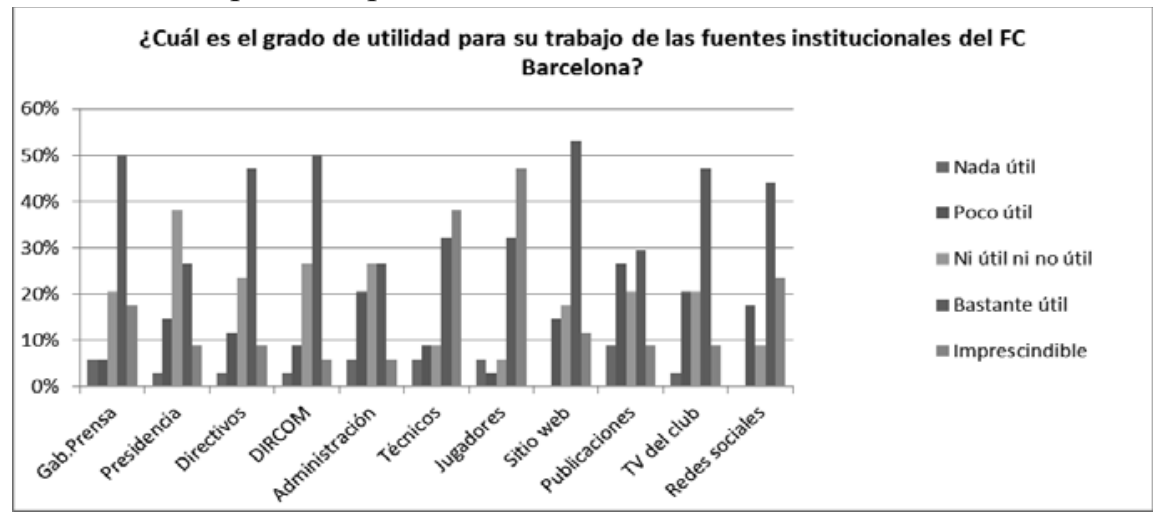

Gráfico 2. Utilidad para los periodistas de las fuentes institucionales del FC Barcelona.

Fuente: elaboración propia

\subsection{Fuentes no institucionales de los periodistas deportivos}

Si observamos las actitudes de los periodistas que informan sobre el FC Barcelona, advertimos que el $94 \%$ de los periodistas considera a los agentes de los jugadores como fuente; un $88 \%$ se apoya en Twitter como fuente de noticias; un $85 \%$ admite que las emisoras de radio también son fuente de información y un 79\% de los encuestados piensa lo mismo de los medios impresos.

\subsection{Tipo de comunicación que realiza el club}

$\mathrm{Al}$ analizar la percepción de los periodistas deportivos sobre la política de comunicación que desarrolla el FC Barcelona, un 66\% considera que la comunicación del club no tiene en cuenta las necesidades de los periodistas. Un 69\% sostiene, asimismo, que está enfocada a construir la imagen corporativa de la entidad. En este sentido, un $66 \%$ asegura que busca crear un clima de opinión favorable, para un $60 \%$ se dirige fundamentalmente a los socios del club, mientras que otro $60 \%$ opina que dicha comunicación está integrada en la estrategia de marketing que desarrolla la entidad.

Opinión sobre la comunicación que realiza el FC Barcelona
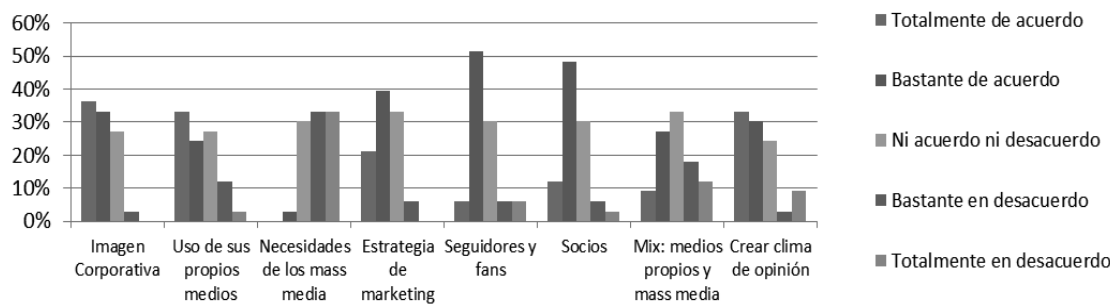

Gráfico 3. Tipo de comunicación que realiza el FC Barcelona, según los periodistas. Fuente: elaboración propia 


\section{a) Valoración de la información que emite el club}

Los periodistas deportivos consideran que el club les envía dos tipos de información: institucional y vinculada a la actualidad deportiva. Por lo que respecta a la información que distribuye el FC Barcelona, un 56\% de los periodistas opina que los contenidos poseen interés informativo. El 53\% estima que el gabinete de prensa no se inmiscuye en su trabajo. El 53\% también considera que emite la información que necesita el periodista y el $65 \%$ opina que es correcta. No obstante, para el $66 \%$ de los encuestados la información que emite el club es parcial, y un $46 \%$ advierte que resulta incompleta. Además, el $31 \%$ de los periodistas llama la atención sobre el hecho de que el gabinete de prensa desconoce las rutinas productivas de los medios.

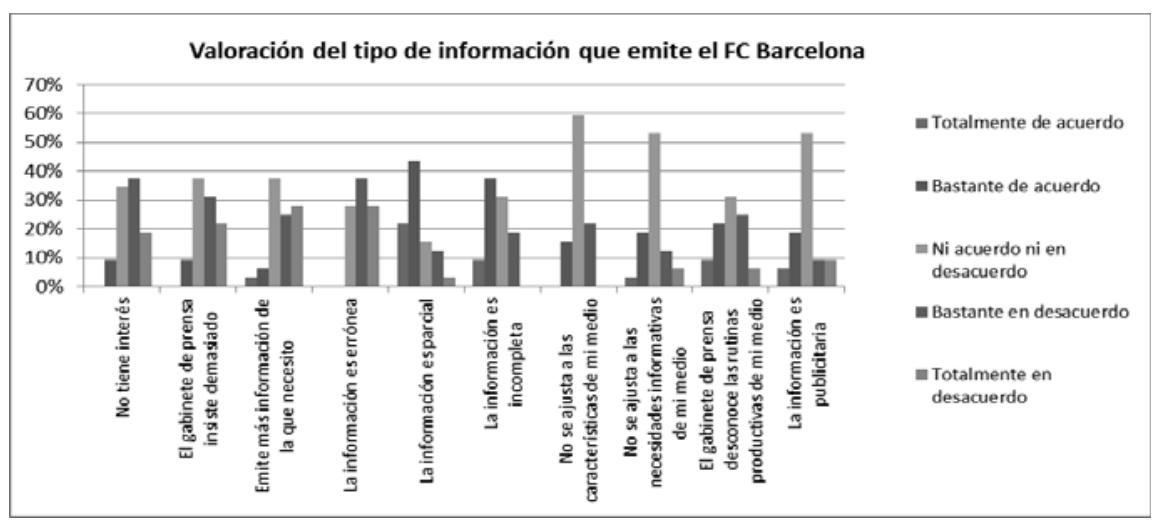

Gráfico 4. Valoración de la información que envía el departamento de Comunicación del FC Barcelona. Fuente: elaboración propia

\section{b) El papel del departamento de Comunicación}

En relación con los datos anteriores, hemos querido analizar las opiniones que los periodistas deportivos tienen sobre el gabinete de Comunicación de la entidad objeto de este estudio. El 54\% de los encuestados considera que el departamento de Comunicación no facilita el trabajo de los periodistas. E1 80\% de los periodistas asegura que la principal función del departamento consiste en filtrar la relación con los jugadores y técnicos del equipo, mientras que un $83 \%$ creen que también desempeña el papel de filtro en las relaciones con los directivos y los periodistas. Para el $84 \%$ de los encuestados, el departamento privilegia a unos medios respecto de otros en la atención informativa y un $81 \%$ opina que el departamento favorece a determinados periodistas con los contenidos que suministra. 


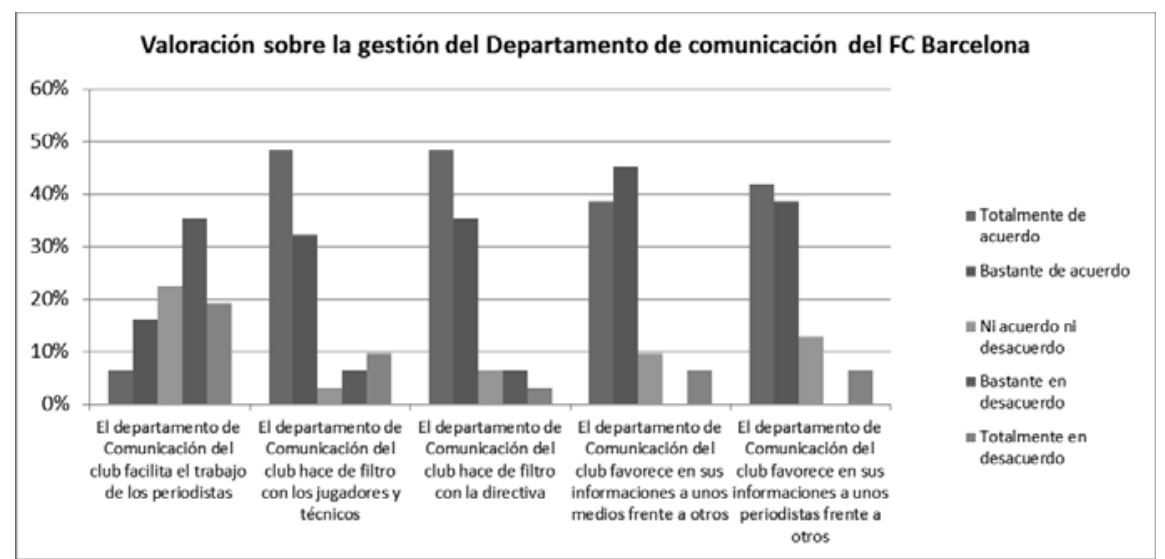

Gráfico 5. Valoración de la gestión del departamento de Comunicación del FC Barcelona. Fuente: elaboración propia

\section{Conclusiones}

El FC Barcelona ha desarrollado un nuevo modelo de gestión comunicativa integral en el que los medios tradicionales ya no desempeñan un papel central dentro de las estrategias comunicativas del club. Un aspecto de la estrategia de comunicación del club catalán es la creación de marca a través de la gestión de contenidos y la dinámica que emplea en su relación con los medios tradicionales como público objetivo. Desde la propia entidad reconocen que ello contribuye decisivamente a potenciar los vínculos emocionales que los seguidores tienen con la organización. Esta reflexión contrasta con la confrontación que existe en la actualidad entre el club y los periodistas deportivos, debido al control que ejerce el departamento de Comunicación.

A medida que ha crecido la visibilidad mediática en paralelo a la dimensión internacional de la institución, su modelo comunicativo se ha configurado en torno a la marca. Como consecuencia, se ha profesionalizado el departamento de Comunicación, se han integrado plataformas comunicativas, se ha originado un mayor control de los mensajes de ambos clubes a través de estos canales de comunicación propios y se ha desplazado el foco de atención informativa del club a los seguidores y socios, en detrimento de los periodistas deportivos. Mayoritariamente, los periodistas encuestados consideran que la política comunicativa que desarrolla el FC Barcelona no está dirigida hacia los medios, ya que no tiene en cuenta las necesidades de los periodistas, al tiempo que entienden que el objetivo del departamento de Comunicación es crear un clima de opinión favorable.

Este comportamiento corrobora que este nuevo modelo implica una transformación de los profesionales del departamento de Comunicación: han pasado de ser meros intermediarios o fuentes especializadas de los periodistas a convertirse en gestores de la información que generan, lo que les permite no sólo difundir los mensajes, sino también controlarlos, de modo más eficaz, a través de sus propios soportes comunicativos. A partir de este cambio, se manifiesta un grado de influencia del club en los productos informativos de los medios, favorecido por el amplio dispositivo comunicativo propio con el que cuenta. 
A la vista de los datos obtenidos, podemos reseñar que, en términos generales, los informadores consideran que el departamento de Comunicación del FC Barcelona se ha convertido en un mecanismo de control y, en consecuencia, las relaciones personales entre ambos actores se han deteriorado, al limitar el acceso a la información. Según los periodistas, este departamento se caracteriza por fiscalizar la información dirigida a los medios y por entorpecer la labor de los informadores.

Por consiguiente, podemos advertir que el modelo comunicativo del FC Barcelona ha modificado la relación con los periodistas que habitualmente cubren la actualidad informativa del club, ya que se limita el acceso directo a las fuentes tradicionales de la información: los jugadores y los entrenadores de los equipos. La bidireccionalidad de la comunicación entre periodistas y profesionales en épocas anteriores, se ha transformado en unidireccionalidad con la gestión comunicativa de estos departamentos.

\section{Referencias bibliográficas}

BADÍA, Jordi (2009): El Barça al descobert. Badalona, Ara Llibres SCCL.

BARROSO, Miguel Ángel (2012): "Estrellas bajo la ley del silencio", en ABC: http://www.abc.es/20120220/deportes/abcp-estrellas-bajo-silencio-20120220.html [Consulta: 10 de septiembre de 2014].

BOYLE, Raymond (2007): "Sports journalism and communication: challenges and opportunities in the digital media age". Asia Communication and Media Forum, 14-16, Sept 2007, Beijing, China.

BOYLE, Raymond (2010): "Sport and the Media in the UK: the Long Revolution?". Sport in Society, vol. 13 (9), pp. 1298-1311, en: http://dx.doi.org/10.1080/17430437 .2010 .510668

BOYLE, Raymond and HAYNES, Richard (2006): "The football industry and the public relations", en Jacquie L'ETANG y Magda PIECZKA (Eds.): Public relations: critical debates and contemporary practice. Mahwah, USA, Lawrence Erlbaum Associates, pp. 221-239.

BOYLE, Raymond and HAYNES, Richard (2009): Power Play. Sport, the Media and Popular Culture. Edinburgh, Edinburgh University Press Ltd.

CLELAND, Jamie (2009): “The Changing Organizational Structure of football clubs and their relationship with external media". International Journal of Sport Communication, vol. 2, pp. 417-431.

CURTIN, Patricia A. (1999): "Reevaluating public relations information subsidies: Marketdriven journalism and agenda-building theory and practice". Journal of Public Relations Research, vol. 11 (1), pp. 53-90.

DESMARAIS, Fabrice and BRUCE, Toni (2008): "Blurring the boundaries of sports public relations: National stereotypes as sport announcers' public relations tools". Public Relations Review, vol. 34 (2), pp. 183-191: http://dx.doi.org/10.1016/j.pubrev.2008.03.016 
FORTUNATO, John A. (2001): "Public relations strategies for creating mass media content: A case study of the National Basketball Association". Public Relations Review, vol. 26 (4), pp. 481-497: http://dx.doi.org/10.1016/S0363-8111(00)00060-6

GIEBER, Walter and JOHNSON, Walter (1961): "The City Hall beat: a study of reporter and source roles". Journalism and Mass Communication Quaterly, vol. 38, pp. 289-297.

GINESTA PORTET, Xavier (2010): "Los medios propios de los clubes de fútbol españoles. De la revista oficial a los canales de TDT". Estudios sobre el mensaje periodístico, vol. 16, pp. 145-166. Madrid, Servicio de Publicaciones de la Universidad Complutense.

GLASER, Barney (1992): Basics of Grounded Theory Analysis. Mill Valley, California, Sociology Press.

GOLAN, Guy and WANTA, Wayne (2001): "Second-level agenda setting in the New Hampshire primary: A comparison of newspaper coverage in three newspapers and public perceptions of candidates". Journalism \& Mass Communication Quarterly, vol. 78 (2), pp. 247-259: http://dx.doi.org/10.1177/107769900107800203

GONZÁLEZ RAMALLAL, Manuel E. (2004): "El reflejo del deporte en los medios de comunicación en España”. Revista de Estudios Sociológicos, vol. 4, pp. 271280.

GONZÁLEZ RAMALLAL, Manuel E. (2014): "Prensa deportiva e identidad nacional: España en el Mundial de fútbol de Sudáfrica 2010". Política y Sociedad, vol. 54, pp. 337-366: http://dx.doi.org/10.5209/rev_POSO.2014.v51.n2.43077

GRANDE, Ildefonso y ABASCAL, Elena (2005). Análisis de encuestas. Madrid, Esic.

HACHIGIAN, David and HALLAHAN, Kirk (2003): "Perceptions of public relations web sites by computer industry journalists". Public Relations Review, vol. 29, pp. 43-62: http://dx.doi.org/10.1016/S0363-8111(02)00195-9

HALLAHAN, Kirk (1999): "Seven models of framing: Implications for public relations". Journal of Public Relations Research, vol. 11 (3), pp. 205-242.

HELLAND, Knut (2007): "Changing Sports, Changing Media. Mass Appeal, the Sports/Media Complex and TV Sports Rights". Nordicom Review, vol. 28, pp. 105119.

HOPCRAFT, Arthur (2013): The Football Man. People and Passions in Soccer. New edition. London, Simon and Schuster.

HOPWOOD, Maria (2007): "It's football but not as you know it: Using Public Relations to promote the world game in Australia". Sport Marketing Europe, $\mathrm{n}^{\mathrm{o}} 3$, pp. 32-37.

HOPWOOD, Maria (2010): "Public Relations and the Media", en HAMIL, Sean y CHADWICK, Simon (Eds): Managing Football. An international perspective. Oxford, Butterworth-Heinemann, pp. 55-57. 
HORKY, Thomas (2009): "Contenidos y modelos de elaboración de la información deportiva en la prensa escrita. Resultados de un estudio comparativo internacional". Apunts. Educación Física y Deportes, $3^{\circ}$ trimestre, pp. 70-79.

JOHNSON AVERY, Elisabeth and WEAVER LARISCY, Ruthann (2007): "Public Relations Practitioners' Relationships with Media and Each Other as Moderators of Excellent Health Information and the Local Public Health Agenda". Public Relations Journal, vol. 1 (1), pp. 1-22.

KUNTZ, Reinhard, WORATSCHECK, Herbert and STRELOW, Marcel (2010): "Future Opportunities and Threats in a Fragmented Sport Media World-Trends and Strategic Implications for Sport Media Enterprises”. Diskussionspapier 09-10, University of Bayreuth, pp. 1-45.

LEDINGHAM, John A. (2003): Explicating relationship management as a general theory of public relations. Journal of Public Relations Research, vol. 15 (2), pp. 181-198: http://dx.doi.org/10.1207/S1532754XJPRR1502_4

LEVER, Janet and WHEELER, Stanton (1993): "Mass media and the experience of sport". Communication Research, vol. 20 (1), pp. 125-143: http://dx.doi.org /10.1177/009365093020001006

LYDENBERG, Steven (2005): Corporations and the public interest: guiding the invisible hand. San Francisco, Berrett-Koehler Publishers.

MANNING, Paul (2001): News and News Sources: A critical introduction. London, Sage.

MATILLA, Kathy (2010): "Pasado, presente y futuro del Dircom en España”. FISECEstrategias, vol. 5 (14), pp. 3-24.

MICÓ-SANZ, Josep Lluís y GONZÁLEZ-MOLINA, Sonia (2010): "Procesos de convergencia en la comunicación empresarial. El caso de las oficinas de prensa especializadas en tráfico y seguridad vial". El profesional de la información, vol. 19 (2), pp. 133-139: http://dx.doi.org/10.3145/epi.2010.mar.03

MORAGAS, Miquel de; KENNETT, Chris; y GINESTA, Xavier (2011): "Football and media in Europe. A new sport paradigma for the global era", en TOMLINSON, Alan; YOUNG, Christopher; \& HOLT, Richard (Eds): Sport and the Transformation of Modern Europe. States, media and markets 1950-2010. Oxon (Reino Unido), Routledge, pp. 128-149.

ORDEIX, Enric y GINESTA, Xavier (2009): “Aplicación de los parámetros de la excelencia en la gestión de relaciones con los medios de comunicación: una comparativa entre FC Barcelona y RCD Espanyol”, en CARRETÓN BALLESTER, María del Carmen (Coord.): La gestión de las relaciones con los públicos. Alicante, Asociación de Investigadores en Relaciones Públicas (AIRP), pp. 102-118.

POST, James; PRESTON, Lee and SACHS, Sybille (2002): "Managing the extended enterprise: the new stakeholder view". California Management Review, vol. 45 (1), pp. 6-28. 
PRUDKIN, Gonzalo (2010): "Blogosfera, Midiaesfera y Fútbol: aspectos del funcionamiento de un nuevo ecosistema informativo en la Argentina", en PRISMA.COM, 9, pp, 1-22. [Consulta: 1 de octubre de 2014]: http://prisma.cetac.up.pt /Prisma.Com_n9_Blogosfera_Midiaesfera_Futbol.pdf

RAMÍREZ, Txema (1995a): Gabinetes de comunicación. Funciones, disfunciones e incidencia. Barcelona, Bosch Comunicación.

RAMÍREZ, Txema (1995b): "La influencia de los gabinetes de prensa. Las rutinas periodísticas al servicio del poder". Revista Telos, no 40, pp. 47-57.

RODRÍGUEZ ANDRÉS, Roberto (2003): "Características socio-demográficas y laborales de los periodistas españoles e índice de satisfacción profesional”. Ámbitos, $\mathrm{n}^{\circ}$ especial 9-10, pp. 487-504.

ROWE, David (1996): "The global love-match: Sport and television”. Media, Culture \& Society, vol. 18 (4), pp. 565-583.

ROWE, David (2004): Sport, Culture and the Media: The Unruly Trinity. Maidenhead, Open University Press.

SALLOT, Lynne y JOHNSON, Elizabeth (2006): "Investigating relationships between journalists and public relations practitioners: Working together to set, frame and build the public agenda, 1991-2004". Public Relations Review, vol. 32 (2), pp. 151-159: http://dx.doi.org/10.1016/j.pubrev.2006.02.008

SANAHUJA PERIS, Guillermo (2013): "Planificación y estrategia de identidad corporativa en los clubes deportivos españoles". Questiones publicitarias, vol. I, núm. 18, pp. 126-143.

SANTOS FERNÁNDEZ, Alex (2010): L'Entorn. El circ mediàtic del Barça. Valls, Cossetània Edicions.

SCHULTZ, Brad y SHEFFER, Mary Lou (2010): “An exploratory study of how Twitter is affecting sports journalism". International Journal of Sport Communication, vol. 3 (2), pp. 226-239.

STOLDT, Clayton; DITTMORE, Stephen; y PEDERSEN, Paul (2011): "Public relations in the sport industry", en PEDERSEN, Paul; PARKS, Janet; QUARTERMAN, Jerome; \& Lucie THIBAULT (Eds.): Contemporary Sport Management. Champaign, IL: Human Kinetics, pp. 270-289.

SUGDEN, John y TOMLINSON, Alan (2007): "Stories from planet football and sportsworld. Source relations and collusion in sport journalism". Journalism Practice, vol. 1 (1), pp. 44-61: http://dx.doi.org/10.1080/17512780601078860

SUMMERS, Jane and MORGAN, Melissa (2008): "More than just the media: Considering the role of public relations in the creation of sporting celebrity and the management of fan expectations". Public Relations Review, vol. 34 (2), pp. 176182: http://dx.doi.org/10.1016/j.pubrev.2008.03.014

TILLEY, Elspeth and HOLLINGS, James (2008): "Still stuck in 'A love-hate relationship': Understanding journalists' enduring and impassioned duality towards 
public relations". ANZCA08 Conference, Power and Place. Wellington, July, pp. $1-24$.

TOMLINSON, Alan and SUGDEN, John (2008): "Sport journalism: persistent themes and changing times", en FRANKLIN, Bob (Ed): Pulling newspapers apart: analysing print journalism. New York, Routledge, pp. 145-153.

TROSBY, Erik (2010): "Public relations, football and the management of player transgressions in Australia". Public Communication Review, vol. 1 (2), pp. 49-66.

VAN RIEL, Cees (1995): Principles of Corporate Communication. London, Prentice Hall.

VINNAI, Gerhard (1986): El fútbol como ideología. México, Siglo XXI Editores.

WHITE, Jon and HOBSBAWN, Julia (2007): "Public Relations and Journalism. The unquiet relationship. A view from the United Kingdom". Journalism Practice, vol. 1 (2), pp. 283-292: http://dx.doi.org/10.1080/17512780701275606

WIGLEY, Shelley and MEIRICK, Patrick (2008): "Interactive Media and Sports Journalists The Impact of Interactive Media on Sports Journalists". Journal of Sports Media, vol. 3 (1), pp. 1-25, http://dx.doi.org/10.1353/jsm.2008.0003

Fernando Olabe Sánchez es doctor en Comunicación y profesor asociado del Departamento de Ciencias Sociales y Humanidades de la Universidad Miguel Hérnández de Elche. Imparte la asignatura Comunicación Corporativa e Institucional 